\title{
Prototipo Caja de Arena de Realidad Aumentada GS-Sandbox
}

\author{
Viracucha Quinga, Edwin Guillermo $^{\text {1,* iD); Manosalvas Granja, Samy Patricio }}{ }^{1}$ (D) \\ ${ }^{1}$ Escuela Politécnica Nacional, Instituto Geofísico, Quito, Ecuador
}

\begin{abstract}
Resumen: A finales del año 2017, se empezó el desarrollo de un prototipo de caja de arena denominado "GSSandbox", iniciativa motivada por revisar técnicas elaboradas en museos interactivos como en Japón, sitios donde se han implementado las técnicas de Realidad Aumentada para concientizar a los ciudadanos japoneses acerca de los fenómenos naturales que se registran año tras año. El objetivo de este estudio es replicar un dispositivo de modelamiento topográfico de realidad aumentada (AR) sobre una caja de arena. Esta será una herramienta interactiva para concientizar a estudiantes de escuelas, colegios, comunidad y entidades gubernamentales acerca de los peligros de los fenómenos naturales, principalmente los que están relacionados con las erupciones volcánicas en el Ecuador. La realidad aumentada se produce al superponer elementos virtuales sobre elementos reales y al utilizar la tecnología informática puede ser visualizado en una pantalla o proyectado al mundo real.
\end{abstract}

Palabras clave: GS-Sandbox, Realidad Aumentada, Curvas de nivel, Volcanes, Realidad Virtual, Lava.

\section{GS-Sandbox Augmented Reality Prototype}

\begin{abstract}
At the end of 2017, the development of a sandbox prototype called "GS-Sandbox" began, an initiative motivated by reviewing techniques developed in interactive museums such as in Japan, sites where Augmented Reality techniques have been implemented to raise awareness among Japanese citizens about natural phenomena that are recorded year after year. The objective of this study is to replicate an augmented reality (AR) topographic modeling device on a sandbox. It will be an interactive tool to raise awareness among students from schools, colleges, community and governmental entities about the dangers of natural phenomena, mainly those related to volcanic eruptions in Ecuador. Augmented reality is produced by superimposing virtual elements on real elements and by using computer technology, it can be visualized on a screen or projected to the real world.
\end{abstract}

Keywords: GS-Sandbox, Augmented Reality, Contours, Volcanoes, Virtual Reality, Lava.

\section{INTRODUCCIÓN}

La realidad aumentada (AR) es un conjunto de dispositivos que añaden información virtual a la información física existente. Es decir, el prototipo GS-Sandbox permite combinar y moldear un entorno físico del mundo real (al mezclar agua con arena) con elementos virtuales (proyección de un mapa topográfico a color y curvas de nivel) que se ajustan con la topografía antes moldeada. La AR, se logra mediante el uso de una cámara $3 \mathrm{D}$, un computador, una tarjeta de video High Definition (HD), un proyector de corto alcance y una superficie moldeable donde se proyecte la imagen.

GS-Sandbox reconoce y modela en tiempo real el relieve y/o cambio en éste, además permite emular fluidos como agua y lava, aplicando las ecuaciones de Navier-Stokes (Reed et al.,
2016), relacionadas a la mecánica de fluidos, mismas que permiten observar su comportamiento en la superficie. Esta es una herramienta de realidad aumentada y se basa en estudios anteriores que permitieron realizar la instalación del software, integración del sistema, configuración, calibración y ejecución del prototipo.

En el año 2014, ARSandBox fue originalmente desarrollada por investigadores de la Universidad de California de Estados Unidos, a partir de una alianza en UC Davis W.M. Keck Center for Active Visualization in the Earth Sciences, junto con UC Davis Tahoe Environmental Research Center, Lawrence Hall of Science y ECHO Lake Aquarium and Science Center. ARSandBox ha sido replicada en todo el mundo, como una herramienta útil para la formación de profesionales y la difusión de las ciencias de la Tierra (Reed et al., 2014). 
En el año 2015, con respecto a Augmented Reality Sandbox, Rick Wilkinson indica que observó ARSandBox de realidad aumentada por primera vez en la página web de UC Davis de Oliver Kreylos y pensó que implementarla sería bastante sencillo. Realizó una prueba de concepto con una computadora Pentium de doble núcleo y tarjeta gráfica, un proyector y un Xbox Kinect prestado. Posterior a instalar todo el software necesario, la computadora funcionaba y generaba la simulación sobre la caja de arena por pocos segundos y luego dejaba de funcionar. Una vez terminada la prueba de concepto y con el presupuesto necesario para el proyecto, empezó a comprar los equipos de acuerdo a los sugeridos por los creadores del ARSandbox. Realizó algunos prototipos de caja de arena, sobre todo porque tenía que considerar el costo, las seguridades y la facilidad para desmontar. Lo que más tiempo le tomó fue el aprender los comandos de Linux y las calibraciones que se debían realizar a los equipos. Finalmente, en noviembre de 2015, pudo concluir con el proyecto por un valor de 800 USD, mismo que se encuentra en un espacio permanente en Innovations Academy (Wilkinson, 2015).

Leytzher Muro, en el año 2019, a necesidad de presentar un proyecto en la Feria de Ciencias, llevó a cabo la construcción de ARSandbox documentada por Oliver Kreylos en su publicación de 2012. Muro, en su resultado final confirmó la exactitud de las medidas de la caja de arena (40"x30" $\mathrm{x} 4$ "), manifestó el ajuste realizado para transformar un proyector convencional a uno de tiro corto usando un espejo, para esto colocó el espejo a un metro de distancia sobre la caja de arena permitiendo así conseguir una imagen de 40"x 30 " y el proyector lo ubicó en el piso en modo "mirror mode", además indicó la necesidad de una tarjeta de video externa si se desea simular lluvia (Muro, 2019).

GS-Sandbox apoyará a la divulgación de información relacionada a los fenómenos naturales: erupciones volcánicas e inundaciones, de una manera interactiva a la comunidad.

En los años 2017 (IGEPN 2017, p. 21) y 2018 (IGEPN 2018, p. 18), el Instituto Geofísico recibió 36 visitas técnicas al centro de monitoreo TERRAS, las cuales representan actividades externas que permiten la divulgación de información sísmica y volcánica hacia la comunidad, la institución actualmente no dispone de una herramienta interactiva que facilite la socialización de la información sobre los peligros asociados a los volcanes.

\section{PROCESO GS-Sandbox}

A continuación, se describe el conjunto de actividades que se deben realizar para la implementación del prototipo GSSandbox mismas que están descritas en la Figura 1.

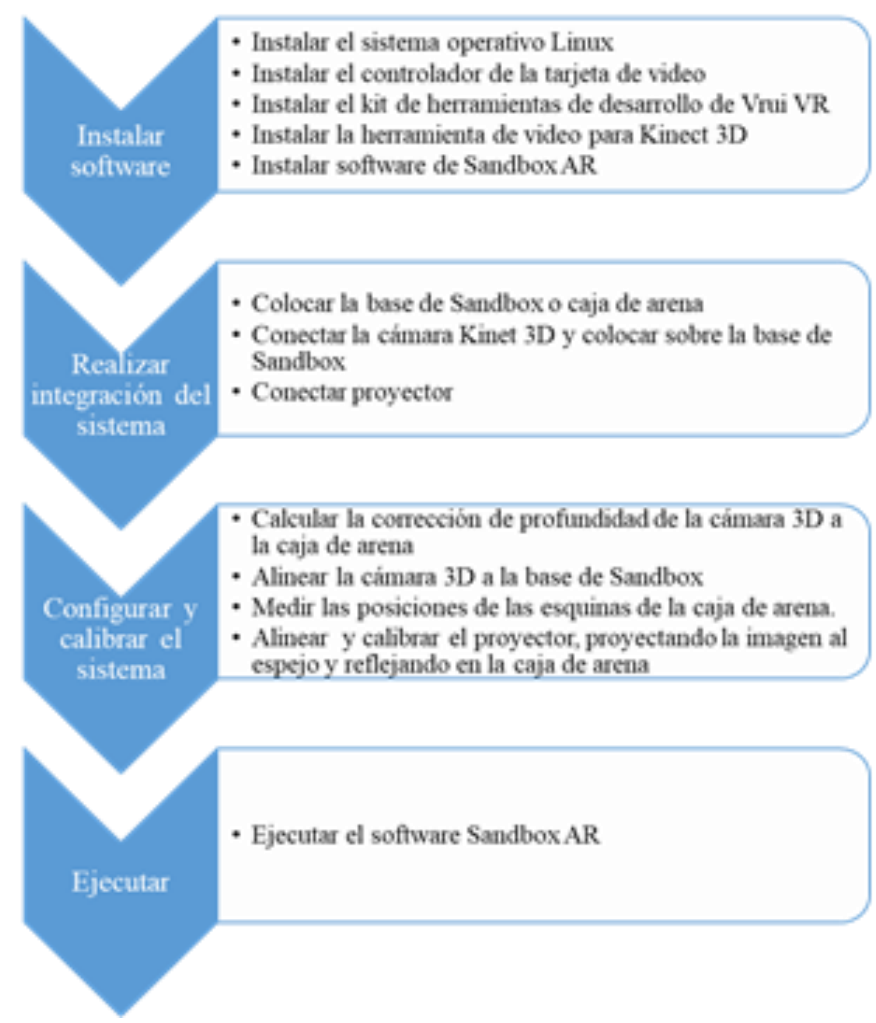

Figura 1. Proceso GS-Sandbox

\section{PROTOTIPO GS-Sandbox}

GS-Sandbox está basado en la documentación citada en los antecedentes, donde el equipo de desarrolladores de los diferentes estudios da a conocer las especificaciones y recomendaciones a tomar en cuenta tanto en hardware como en software para llevar a cabo la réplica y el desarrollo del ARSandbox. Sin embargo, esté prototipo, al no contar con los recursos necesarios, se adaptó algunos de los componentes a la disponibilidad y recursos económicos de los autores, lo cual, al momento de la implementación del GS-Sandbox no causó inconvenientes.

GS-Sandbox está compuesto de seis partes en hardware: a) un computador de escritorio con tarjeta de video, b) un sensor Kinect y adaptador USB a 2.0, c) un proyector, d) un espejo, e) caja de arena, f) una estructura de metal y madera, y una parte en software: g) software de código libre.

\subsection{Requerimientos de Hardware}

a) Computador de escritorio con tarjeta de video: para un correcto funcionamiento del software, es necesario tomar en cuenta las exigencias expresadas por Oliver Kreylos en su portal web https://web.cs.ucdavis.edu. Para el prototipo, se utilizó un computador HP COMPAQ dc5800 PC Intel® Core $^{\mathrm{TM}} 2$ Duo E8400, una tarjeta de memoria RAM de 4 GB DDR2-SDRAM, un disco duro de 500GB de almacenamiento, una tarjeta de video NVIDIA GeForce 9400GT y sistema operativo Linux Mint. Es importante contar con el procesador y la tarjeta de video especificados, ya que estos componentes permiten la generación del mapa topográfico y la simulación de flujos de agua o lava de una manera óptima. 
b) Sensor Kinect y adaptador USB a 2.0: es una cámara de profundidad y con su percepción en 3D permite capturar los movimientos de las personas de manera eficiente, mientras que la cámara de infrarrojos permite diferenciar la profundidad. El adaptador USB a 2.0 permite la conexión del Kinect 3D a la computadora a través del puerto USB, este recopila información espacial del relieve modelado en la caja de arena para ser procesado por el software ARSandbox y ser proyectada sobre la superficie moldeada.

c) Proyector: el modelo a usar es BENQ MS517 con las características: brillo de 2800 Lúmenes, relación de Contraste de 13000:1, resolución SVGA, puertos HDMI y VGA; que permite proyectar e interactuar con el mapa topográfico generado por el software ARSandbox, sobre la superficie de la topografía moldeada con la arena.

d) Espejo: para transformar el proyector convencional a uno de tiro corto se coloca un espejo de cinco líneas de espesor y una dimensión de $30^{\prime \prime} \times 30^{\prime \prime}$ a un metro de altura de la caja de arena, con un ángulo regulable $<10^{\circ}$.

e) Caja de Arena: en la Figura 2, se muestra la caja de arena que está elaborada de espuma Flex de un espesor de $4 \mathrm{~cm}$ y tiene una relación de aspecto de 40"x30" para que coincida con los campos de visión del sensor Kinect y el proyector. La caja de arena se llena con arena artificial a un espesor máximo de $10 \mathrm{~cm}$, tiene un peso aproximado de $98 \mathrm{Kg}$ y se recomienda mantener la arena ligeramente húmeda para que sea moldeable.

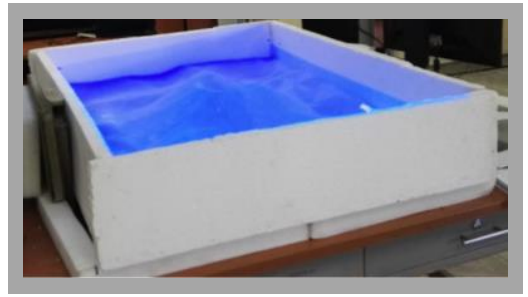

Figura 2. Caja de Arena de 40 "x30"

f) Estructura de metal y madera: se ha elaborado la estructura metálica con cuatro mástiles distribuidos a sus costados con una altura de $110 \mathrm{~cm} \times 170 \mathrm{~cm}$, la cual permite montar el sensor kinect y el espejo, además de la estructura de madera que permite colocar sobre ella el proyector y la caja de arena, como se muestra en la Figura 3.

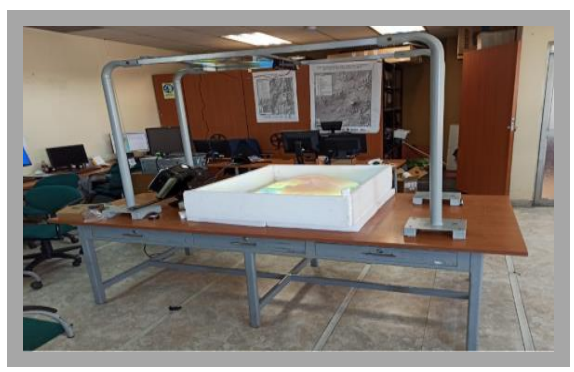

Figura 3. Estructura de metal y madera

Otros periféricos necesarios: cortapicos, canaletas y una extensión de $4 \mathrm{~m}$ de cable (HDMI o VGA) para conectar la salida de video del computador al proyector.

\subsection{Requerimientos de Software}

g) Software de código libre: Dentro del paquete ARSAndbox en su manual de instalación especifica los paquetes de software que se deben instalar, mismos que se distribuyen libremente bajo licencia GNU General Public License versión 2 en el portal web https://web.cs.ucdavis.edu/ okreylos/ResDev/SARndbox/Sof twareInstallation.html.

Para el prototipo GS-Sandbox se instaló:

- Linux Mint 19.3 con MATE desktop environment, 64-bit version.

- Version 5.2-001 del Vrui VR Development Toolkit (automatically selected by Vrui installation script)

- Version 3.9 del Kinect 3D Video Package

- Version 2.7 del Augmented Reality Sandbox

\section{OPERACIÓN GS-Sandbox}

A continuación, la operación de ensamblado del prototipo:

a) Para iniciar con el funcionamiento del prototipo se debe armar la estructura metálica, luego colocar el espejo, el sensor Kinect sobre las dos barras metálicas y de base la caja de arena.

b) Posterior se debe realizar el centrado al sensor Kinect para que se encuentre ubicado en el centro de la caja de arena.

c) Ubicar el proyector apoyado a la caja de arena proyectando la imagen al espejo que se encuentra en la parte superior.

d) Incorporar la arena artificial dentro de la caja de arena alrededor de $30 \mathrm{~cm}$ de espesor y con ayuda de una regla se aplana la superficie para que todo quede uniforme y calibrado.

e) Realizar la alimentación de energía eléctrica a la computadora de escritorio, proyector y Kinect.

f) Los dispositivos como el sensor Kinect y el proyector son conectados a los puertos de la tarjeta gráfica de la computadora.

GS-Sandbox es transportable a cualquier sitio donde se vaya a realizar la capacitación.

A continuación, la operación del software SARndbox.:

a) Encender la computadora con el sistema operativo Linux Mint.

b) Abrir una terminal y buscar el directorio donde se instaló el SARndbox-2.7.

c) Ejecutar el comando RunSARndbox.sh para iniciar la aplicación.

d) Una vez iniciado el programa, se empieza a visualizar las imágenes en la caja de arena como son los colores y las líneas topográficas proyectadas a través del proyector.

e) Con las manos se procede a moldear la arena artificial formando montañas o lagos y el sensor Kinect irá capturando los datos en tiempo real del cambio de pendiente de la arena artificial; la computadora a su vez mediante el software irá reprocesando la información y actualizando las imágenes proyectadas sobre la caja de arena. 


\section{RESULTADOS DEL PROTOTIPO}

El resultado de la implementación del prototipo GS-Sandbox se muestra en la Figura 4, donde se puede observar la caja de arena de realidad aumentada, constituido por cuatro mástiles metálicos, unidos por dos barras de aluminio las cuales sirven de soporte para colocar el sensor Kinect y el espejo. Adicionalmente, se aprecia la caja de arena con su respectiva arena artificial, que sirve como un lienzo para visualizar la imagen a través de la proyección de los mapas generados por el software.

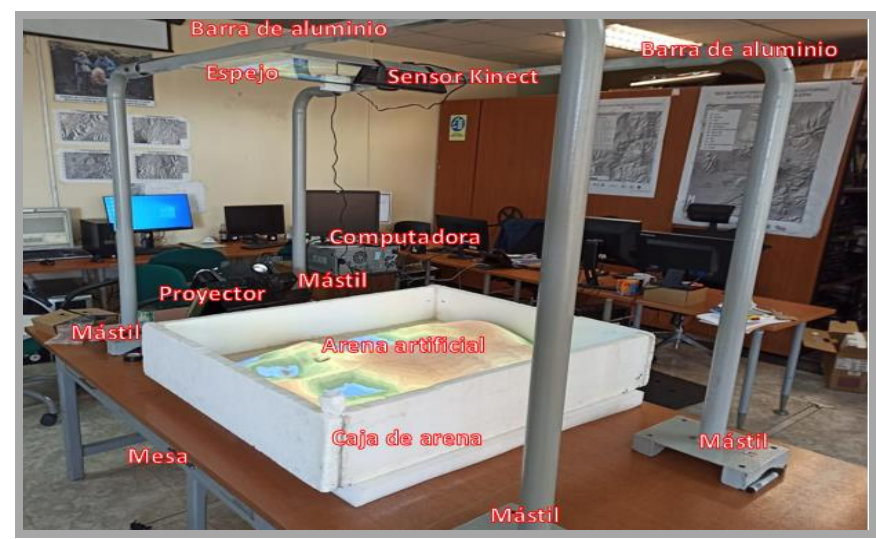

Figura 4. Prototipo GS-Sandbox

Para obtener un resultado óptimo en la proyección de la topografía sobre la caja de arena, se debe realizar el centrado del sensor Kinect seguido del espejo, permitiendo así proyectar la imagen de manera adecuada y cubrir completamente la caja de arena como se muestra en la Figura 5.

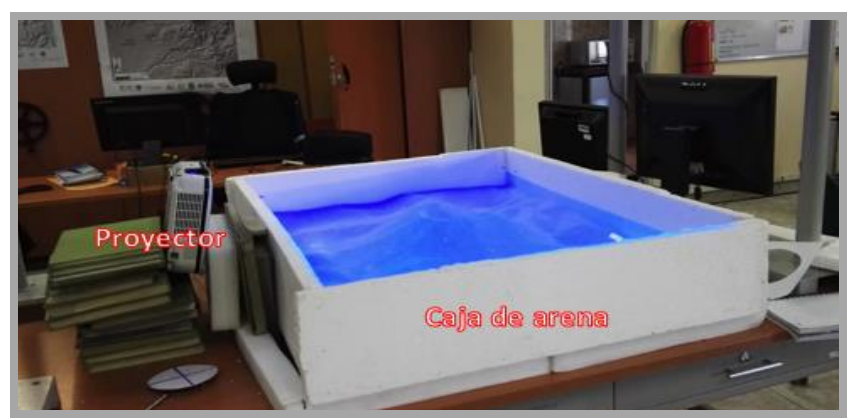

Figura 5. Centrado de sensor Kinect, espejo y proyección de Imagen

Para replicar la topografía de un volcán, se debe moldear la arena artificial dándole forma con nuestra creatividad, el sensor Kinect adquiere los datos de distancia respecto a la caja de arena y estos se envían al programa informático, que, a su vez, va cambiando la proyección de la topografía en tiempo real. En la Figura 6, se puede observar la topografía del relieve moldeado que representa un volcán, la paleta de colores: el color blanco representa la parte más alta del relieve (cumbres) y el color azul representa la parte más baja (nivel del mar). Las líneas de color negro representan las curvas de nivel.

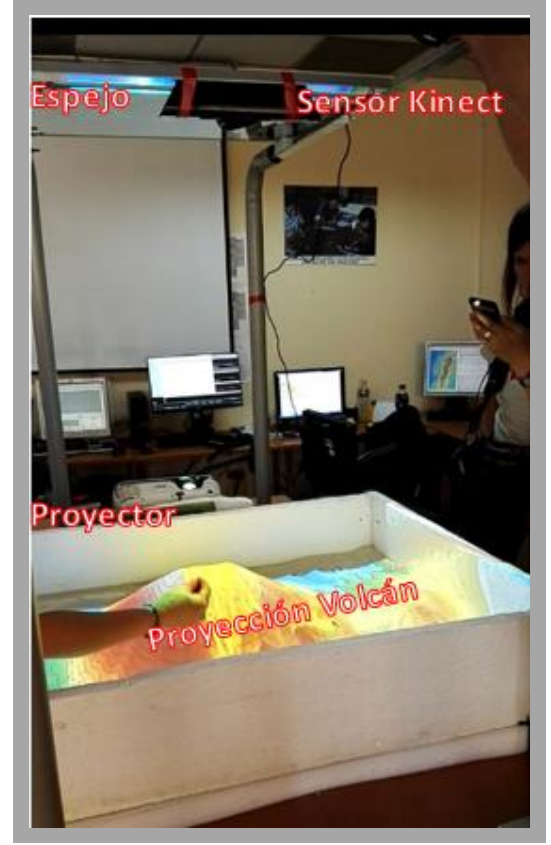

Figura 6. Proyección topografía

Para la funcionalidad del modelado de fluidos y activar la generación de lava, se debe modificar la configuración del programa ARSandbox desactivando la opción de generar lluvia. Una vez realizado este cambio en la configuración del programa, se debe guardar las modificaciones y recompilar la aplicación. Para que el sensor Kinect active la funcionalidad de fluidos, se debe posicionar la mano entre la caja de arena y el sensor. Se ejecuta nuevamente el programa y en la Figura 7 se muestra un volcán con lava en su interior de esta manera se representa una erupción volcánica de tipo hawaiana (López-Ruiz \& Cebriá, 2007,p. 13), como se producen en los volcanes de Galápagos o Hawái.

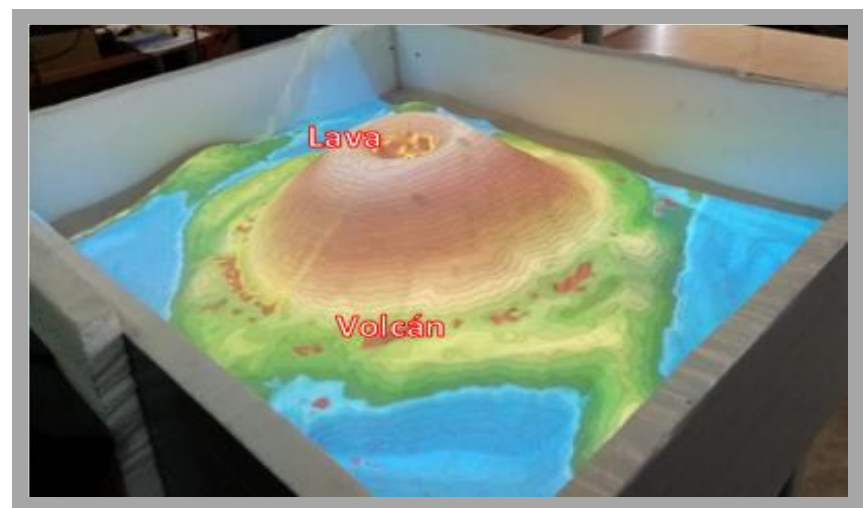

Figura 7. GS-Sandbox, simulación de erupción volcánica tipo Hawaiana

En la Figura 8, se muestra el procedimiento para realizar la simulación de una inundación con lluvia: a) Moldeado, b) Configuración lluvia y c) Proyección. 


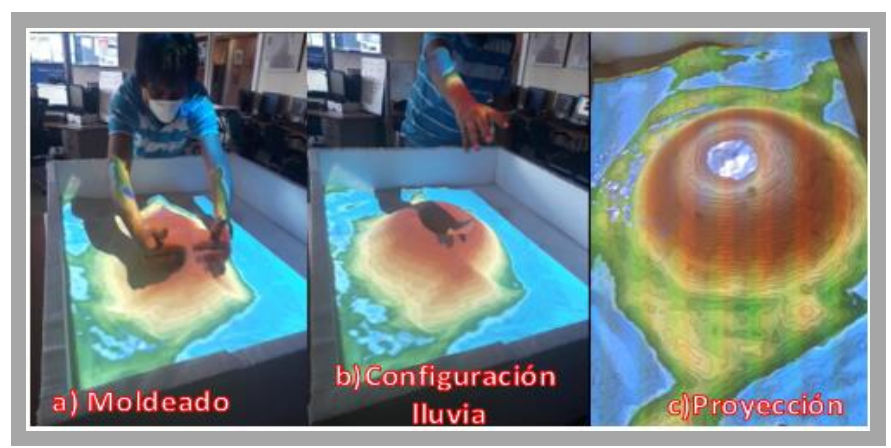

Figura 8. GS-Sandbox, simulación de inundación por lluvia.

Como parte de los resultados se registra la presentación del prototipo en dos eventos para la comunidad, que fueron:

\section{a) Curso vacacional denominado "Planeta Tierra: más allá} de tus ojos"

Una vez realizadas las pruebas del prototipo GS-Sandbox, en el año 2019 se tiene la oportunidad de mostrar el mismo a un grupo de niños y jóvenes que participaron en el curso organizado por Instituto Geofísico (IG) en colaboración con Tía SA y la Escuela Fe y Alegría (Hidalgo, 2019). Como parte del curso, el grupo de niños y jóvenes asistió a una visita guiada al Centro de monitoreo TERRAS del Instituto Geofísico, donde conocieron cómo se realiza el monitoreo sísmico y volcánico en el Ecuador. El área de vulcanología del IG estaba a cargo de la visita guiada y solicitó a los autores del prototipo GS-Sandbox se mostrara su funcionamiento a los niños y jóvenes que participaban de este curso. Se impartió una pequeña charla informativa donde pudieron conocer: los componentes con los que está constituido el GS-Sandbox y el funcionamiento del software y hardware que permite la adquisición de datos y la proyección de la simulación. Una vez finalizada la explicación y con ayuda de los niños se procedió a manipular la arena, dándole la forma de un volcán. El sensor Kinect realizó la adquisición de los datos, después el programa informático generó el modelo y finalmente se proyectó la simulación en la caja de arena como se muestra en la Figura 9. Para finalizar se preguntó a los niños y jóvenes ¿cómo les había parecido esta herramienta?, ellos indicaron que es una herramienta novedosa e interesante por la parte interactiva; era la primera vez que veían algo así.

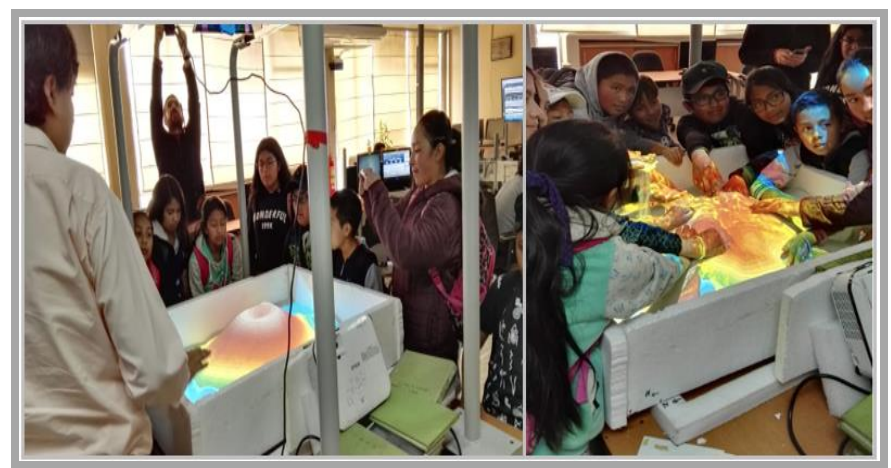

Figura 9. Niños y jóvenes que participaron en el curso vacacional "Planeta Tierra: más allá de tus ojos" b) El Instituto Geofísico participo en la "Expo Feria Espacios de Ciencia para la Paz y el Desarrollo"

En noviembre de 2019, el Instituto Geofísico participó en la "Expo Feria Espacios de Ciencia para la Paz y el Desarrollo" (Viracucha, 2019), la cual se realizó en el Museo Interactivo de Ciencia (MIC), donde, se expuso los diferentes equipos, técnicas y herramientas informáticas que se utilizan para el monitoreo y procesamiento de la información sísmica y volcánica, así como también los canales que utiliza el IG para la difusión de esta información. En la Figura 10 se observa la interacción de la comunidad con el GS-Sandbox, esta tuvo una acogida positiva durante las visitas donde se contó con la participación de niños, jóvenes, adultos y profesionales, así como también de profesores de colegios y universidades, quienes indicaron que la forma de proyectar la información en la caja de arena y la manipulación es bastante interesante ya que de esta manera facilita el aprendizaje sobre los fenómenos naturales como las erupciones volcánicas o inundaciones. Además, personal del MIC manifestó que esta herramienta es interesante y preguntaron cómo podían implementar la misma en el museo.

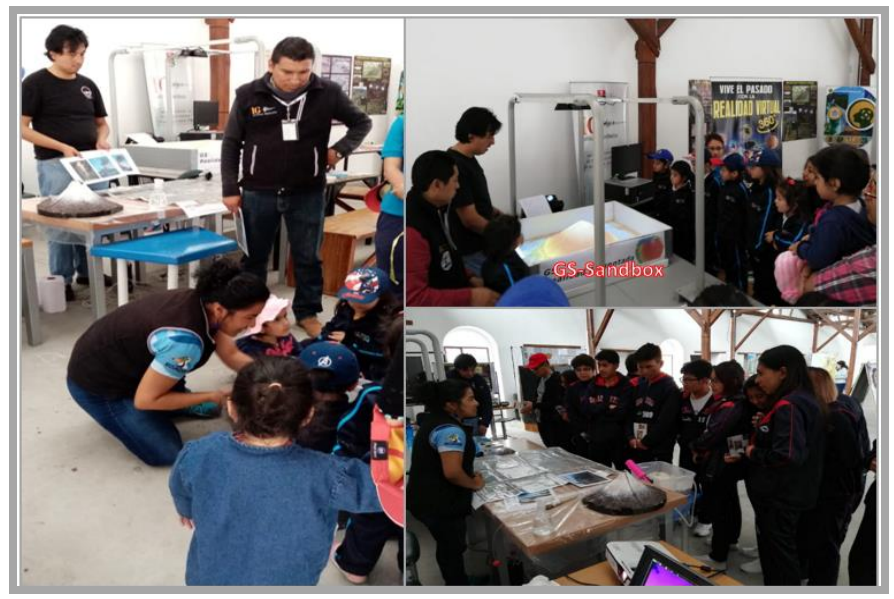

Figura 10. Personal del Instituto Geofísico en el Museo Interactivo de Ciencia (MIC), compartiendo información con niños, jóvenes y adultos

\section{CONCLUSIONES}

Con la construcción del prototipo GS-Sandbox, se demostró que es posible la realización de esta herramienta de realidad aumentada con recursos que pueda contar la institución o las personas interesadas.

Con la participación del GS-Sandbox en el expo feria y el curso vacacional, se pudo evidenciar la aceptación positiva por parte de la comunidad conformada por niños, jóvenes, adultos y profesionales. Por lo que es importante que el Instituto Geofísico asigne presupuesto para construir una versión final del GS-Sandbox.

Con la construcción del prototipo GS-Sandbox, se evidenció que se puede realizar ajustes a la caja de arena y a la estructura metálica y de madera para facilitar su transportación y disminuir su tamaño.

El proyector que se utilizó en el prototipo GS-Sandbox permitió conocer la configuración que se debe realizar al momento de la proyección en la caja de arena. Es importante 
contar con la tarjeta de video HD, para que los gráficos y las simulaciones se ejecuten de forma rápida y óptima, concluyendo así que para obtener una mayor resolución es necesario contar con una tarjeta externa y un proyector de alta resolución.

Para evitar efectos secundarios a las vías respiratorias y alergias a la piel, es necesario que, para el uso del prototipo GS-Sandbox, se utilice arena artificial de juego ya que no contiene ni libera polvo fino de sílice.

\section{REFERENCIAS}

Hidalgo, V. (2019, agosto 13). Vacacional Sierra 2019: Planeta Tierra, Más Allá De Tus Ojos. https://www.feyalegria.org.ec/index.php/quehacemos/noticias/291-vacacional-sierra-2019-planetatierra-mas-alla-de-tus-ojos

IGEPN, Instituto Geofísico. (2017). Informe de Gestión 2017 (p. 33) [Rendición de Cuentas]. Instituto Geofísico de la Escuela Politécnica Nacional. https://www.igepn.edu.ec/transparencia/rendicion-decuentas-2017/2017-fase2/20377-informe-rendicion-decuentas-igepn-2017

IGEPN, Instituto Geofísico. (2018). Informe de Gestión 2018 (p. 42) [Rendición de Cuentas]. Instituto Geofísico de la Escuela Politécnica Nacional. https://www.igepn.edu.ec/transparencia/rendicion-decuentas-2018/2018-fase2/22131-informe-rendicion-decuentas-igepn-2018/file

López-Ruiz, J., \& Cebriá, J. M. (2007). Volcanic processes and risk. Estudios Geológicos, 63(2), 41-65. https://doi.org/10.3989/egeol.07632198

Muro, L. (2019, junio 5). Realidad AumentadaConstruyendo un ARSandbox. Medium. https://medium.com/@leytzher/realidad-aumentadaconstruyendo-un-arsandbox-6920258095ff

Reed, S. E., Kreylos, O., Hsi, S., Kellogg, L. H., Schladow, G., Yikilmaz, M. B., Segale, H., Silverman, J., Yalowitz, S., \& Sato, E. (2014). Shaping Watersheds Exhibit: An Interactive, Augmented Reality Sandbox for Advancing Earth Science Education. AGU Fall Meeting Abstracts, 34, ED34A-01.

Reed, S., Hsi, S., Kreylos, O., Yikilmaz, M., Schladow, S., Segale, H., \& Chan, L. (2016). Augmented Reality Turns a Sandbox into a Geoscience Lesson. Eos, 97. https://doi.org/10.1029/2016EO056135

Viracucha, E. (2019). El IGEPN participó en la "Expo Feria Espacios de Ciencia para la Paz y el Desarrollo"Instituto Geofísico-EPN. Obtenido de: https://www.igepn.edu.ec/interactuamos-con-usted/1767el-igepn-participo-en-la-expo-feria-espacios-de-cienciapara-la-paz-y-el-desarrollo
Wilkinson, R. (2015). Augmented Reality Sandbox. https://docplayer.net/22031287-Augmented-realitysandbox.html

\section{BIOGRAFÍAS}

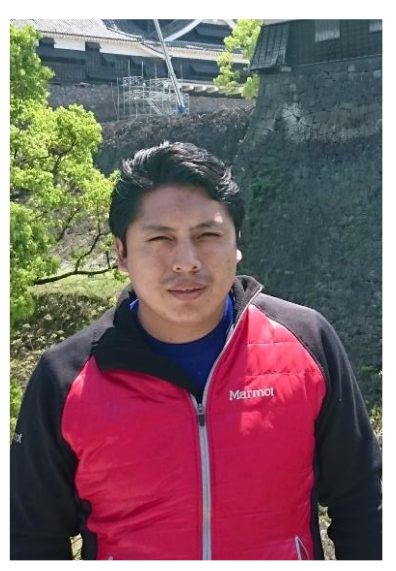

Edwin Guillermo Viracucha Quinga, Ingeniero en Informática, Universidad Tecnología América en el año 2012. Obtuvo su título de Magíster en Disaster Management del National Graduate Institute For Policy Studies (GRIPS) en Japón, en el año 2017, con ayuda de una beca otorgada por el gobierno japonés mediante la Agencia de Cooperación Internacional del Japón (JICA).

Trabaja como Analista de redes de monitoreo, en el Instituto Geofísico de la Escuela Politécnica Nacional. Actualmente se desempeña como coordinador del Centro de Monitoreo TERRAS del Instituto Geofísico.

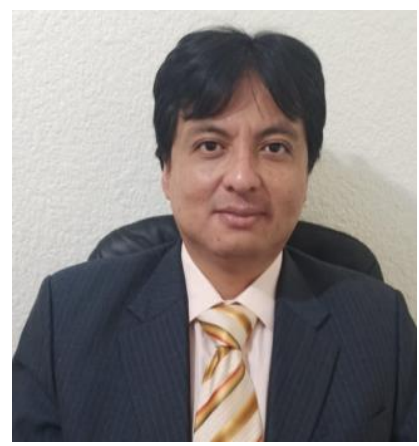

Samy Patricio Manosalvas Granja. Nacido en IbarraEcuador en 1983. Ingeniero en Sistemas Informáticos y Computación (2009) de la Escuela Politécnica Nacional. Master of Science in Geographical Information Science \& Systems (2019) de la Universidad de Salzburgo Austria. Cuenta con certificaciones internacionales vigentes: Agil Coach Professional (ACPC), Scrum Product Owner (SPOPC) y Scrum Master Professional (SMPC). Posee más de 15 años de experiencia laboral para el sector privado y público como director de proyectos, arquitecto y líder de desarrollo de software además de formador en educación virtual. Posee la patente Nro. QUI-056508 por el software "SAM - Sistema de Administración y Monitoreo de Actividad Sísmica y Volcánica". 